Volume 25 (2019) 35-42

DOI: $10.24330 /$ ieja. 504105

\title{
ON ALMOST SUBNORMAL SUBGROUPS AND MAXIMAL SUBGROUPS IN SKEW LINEAR GROUPS
}

\author{
Truong Huu Dung \\ Received: 19 January 2018; Revised: 24 May 2018; Accepted: 24 June 2018 \\ Communicated by A. Çiğdem Özcan

\begin{abstract}
In this paper, we study almost subnormal subgroups and maximal subgroups in skew linear groups satisfying a generalized Laurent polynomial identity.
\end{abstract}

Mathematics Subject Classification (2010): 16K20, 16R40

Keywords: Division ring, linear group, almost subnormal subgroup, maximal subgroup, Laurent polynomial identity

\section{Introduction and preliminaries}

Let $D$ be a division ring with center $F$. Recently, some skew linear groups satisfying an identity was investigated $[4,10,11,12,13]$. For example, in [4] it was

shown that every subnormal subgroup $N$ of $\mathrm{GL}_{n}(D)$ satisfying a generalized group identity over $\mathrm{GL}_{n}(D)$ is central, i.e. $N \subseteq F$, provided $F$ is infinite. Later, in [12] this result was extended for almost subnormal subgroups of $\mathrm{GL}_{n}(D)$. Additionally, L. Makar-Limanov proved that if $D$ is infinite dimensional over its infinite center $F$, then any subnormal subgroup of $D^{*}$ satisfying a generalized Laurent polynomial identity over $D$ is central [11].

Our first aim in this paper is to generalize the above results for almost subnormal subgroups of $\mathrm{GL}_{n}(D)$ satisfying a generalized Laurent polynomial identity in the case when $D$ is algebraic over its uncountable center $F$ and $[D: F]=\infty$. In fact, we prove that if $N$ is an almost subnormal subgroup of $\mathrm{GL}_{n}(D)$ satisfying a generalized Laurent polynomial identity, then $N$ is central (Theorem 2.5). Secondly, we focus on maximal subgroups of $\mathrm{GL}_{n}(D)$ satisfying a Laurent polynomial identity by proving that if $M$ is a maximal subgroup of $\mathrm{GL}_{n}(D)$ such that $D$ is infinite dimensional over its infinite center $F$ and $F[M]$ is algebraic over $F$, then $M$ is absolutely irreducible (Theorem 2.6). This result generalizes partially [10, Theorem 4.1]. In the case when $n=1$, we investigate maximal subgroups of an almost subnormal subgroup of $D^{*}$. In [13], maximal subgroups of subnormal subgroups of $D^{*}$ was studied and it was shown that every nilpotent maximal subgroup of a subnormal subgroup of 
$D^{*}$ is abelian [13, Theorem 2.3]. We extend this result for any maximal subgroup $M$ of a non-central almost subnormal subgroup of $D^{*}$ in the case when $D$ is infinite dimensional over its infinite center $F$ and $C_{D}(M) \backslash F$ contains an algebraic element over $F$. Namely, we show that if $M$ satisfies a Laurent polynomial identity, then $M$ is abelian (Theorem 2.10).

Now, we recall some notation we use in this paper. Let $D$ be a division ring with center $F$ and $G$ be the free group generated by $m$ non-commuting indeterminates $x_{1}, x_{2}, \ldots, x_{m}$. Denote by $\mathrm{M}_{n}(D) *_{F} F G$ the free product of the matrix ring $\mathrm{M}_{n}(D)$ and the group algebra $F G$ over $F$. An element $f\left(x_{1}, x_{2}, \cdots, x_{m}\right) \in \mathrm{M}_{n}(D) *_{F} F G$ is called a generalized Laurent polynomial over $\mathrm{M}_{n}(D)$ (see [5] for the definition of generalized Laurent polynomials over an arbitary algebra). In particular, if $f \in F G$, then $f$ is called a Laurent polynomial over $F$.

Assume that $f\left(x_{1}, x_{2}, \ldots, x_{m}\right)$ is non-zero and $N$ is a subset of the general skew linear group $\mathrm{GL}_{n}(D)$. If $f\left(c_{1}, c_{2}, \ldots, c_{m}\right)=0$ for every $\left(c_{1}, c_{2}, \cdots, c_{m}\right) \in N^{m}$, then we say that $N$ satisfies the generalized Laurent polynomial identity (briefly, GLPI) $f=0$. In this case, $f=0$ is called a generalized Laurent polynomial identity of $N$. Additionally, if $f$ is a Laurent polynomial, then we simply say that $f=0$ is a Laurent polynomial identity of $N$ or $N$ satisfies the Laurent polynomial identity (shortly, LPI) $f=0$.

Let $K$ be a group. Following Hartley [8], $H$ is an almost subnormal subgroup of $K$ if there is a family of subgroups $H=H_{r} \leq H_{r-1} \leq \cdots \leq H_{1}=K$ of $K$ such that for each $1<i \leq r$, either $H_{i}$ is normal in $H_{i-1}$ or $H_{i}$ has finite index in $H_{i-1}$. We call such a series of subgroups an almost normal series of $H$ in $K$. It was noted in [12] that there is a division ring whose multiplicative group contains some almost subnormal subgroup that is not subnormal.

\section{Results}

Let us denote by $\mathrm{M}_{n}(D)\left[t_{1}, t_{2}, \ldots, t_{m}\right]$ the polynomial ring in the determinates $t_{1}, t_{2}, \ldots, t_{m}$ over $\mathrm{M}_{n}(D)$. The following lemma can be obtained by applying the Vandermonde argument [14, Proposition 2.3.26 and 2.3.27].

Lemma 2.1. Let $f\left(t_{1}, t_{2}, \ldots, t_{m}\right) \in \mathrm{M}_{n}(D)\left[t_{1}, t_{2}, \ldots, t_{m}\right]$. If there exist infinitely many elements $\alpha_{1}, \alpha_{2}, \ldots, \alpha_{m}$ in the center $F$ of $D$ such that $f\left(\alpha_{1}, \alpha_{2}, \ldots, \alpha_{m}\right)=0$, then $f$ is identically zero.

Lemma 2.2. Let $D$ be a division ring with infinite center $F$ and $\mathrm{M}_{n}(D)$ be the matrix ring over $D$. If $a \in \mathrm{M}_{n}(D)$ is algebraic over $F$, then there exist infinitely many elements $\alpha \in F$ such that $1+\alpha a \in \mathrm{GL}_{n}(D)$ and 


$$
(1+\alpha a)^{-1}=-\frac{(1+\alpha a)^{k-1}+v_{k-1}(\alpha)(1+\alpha a)^{k-2}+\cdots+v_{1}(\alpha)}{v_{0}(\alpha)},
$$

where $v_{i}(t) \in F[t]$.

Proof. Since $a \in \mathrm{M}_{n}(D)$ is algebraic over $F$, there exists a polynomial

$$
u(x)=x^{k}+b_{k-1} x^{k-1}+\cdots+b_{1} x+b_{0} \in F[x]
$$

such that $u(a)=0$. Let $t$ be a central indeterminate. Put $h_{t}(x)=u\left(\frac{x-1}{t}\right)$.

Then, $h_{t}(x)=\frac{1}{t^{k}}(x-1)^{k}+\frac{b_{k-1}}{t^{k-1}}(x-1)^{k-1}+\cdots+\frac{b_{1}}{t}(x-1)+b_{0}$. Hence

$$
\begin{aligned}
t^{k} h_{t}(x) & =(x-1)^{k}+t b_{k-1}(x-1)^{k-1}+\cdots+t^{k-1} b_{1}(x-1)+t^{k} b_{0} \\
& =x^{k}+v_{k-1}(t) x^{k-1}+\cdots+v_{1}(t) x+v_{0}(t),
\end{aligned}
$$

where $v_{i}(t) \in F[t]$. It is clear that $h_{t}(1+t a)=0$, so

$$
(1+t a)^{k}+v_{k-1}(t)(1+t a)^{k-1}+\cdots+v_{1}(t)(1+t a)+v_{0}(t)=0 .
$$

Since $v_{0}(t)$ has finitely many roots in $F$, there exist infinitely many elements $\alpha \in F$ such that

$$
(1+\alpha a)^{-1}=-\frac{(1+\alpha a)^{k-1}+v_{k-1}(\alpha)(1+\alpha a)^{k-2}+\cdots+v_{1}(\alpha)}{v_{0}(\alpha)} .
$$

This completes the proof.

Let $F\left\langle y_{1}, y_{2}, \ldots, y_{m}\right\rangle$ be the free algebra in $y_{1}, y_{2}, \ldots, y_{m}$ over $F$ and

$$
\mathrm{M}_{n}(D)\left\langle y_{1}, y_{2}, \ldots, y_{m}\right\rangle=\mathrm{M}_{n}(D) *_{F} F\left\langle y_{1}, y_{2}, \ldots, y_{m}\right\rangle
$$

be the free product of $\mathrm{M}_{n}(D)$ and $F\left\langle y_{1}, y_{2}, \ldots, y_{m}\right\rangle$ over $F$. Denote by

$$
\mathrm{M}_{n}(D)\left\langle y_{1}, y_{2}, \ldots, y_{m}\right\rangle\left[\left[t_{1}, t_{2}, \ldots, t_{m}\right]\right]
$$

the ring of formal power series in the indeterminates $t_{1}, t_{2}, \ldots, t_{m}$ with coefficients in $\mathrm{M}_{n}(D)\left\langle y_{1}, y_{2}, \ldots, y_{m}\right\rangle$.

Lemma 2.3. If $f\left(x_{1}, x_{2}, \ldots, x_{m}\right)$ is a non-zero element in $\mathrm{M}_{n}(D) *_{F} F G$, then

$$
f\left(1+t_{1} y_{1}, 1+t_{2} y_{2}, \ldots, 1+t_{m} y_{m}\right)
$$

is a non-zero element in $\mathrm{M}_{n}(D)\left\langle y_{1}, y_{2}, \ldots, y_{m}\right\rangle\left[\left[t_{1}, t_{2}, \ldots, t_{m}\right]\right]$. 
Proof. If

$$
f\left(1+t_{1} y_{1}, 1+t_{2} y_{2}, \ldots, 1+t_{m} y_{m}\right) \equiv 0
$$

in $\mathrm{M}_{n}(D)\left\langle y_{1}, y_{2}, \ldots, y_{m}\right\rangle\left[\left[t_{1}, t_{2}, \ldots, t_{m}\right]\right]$, then

$$
f\left(1+t_{1} \frac{x_{1}-1}{t_{1}}, 1+t_{2} \frac{x_{2}-1}{t_{2}}, \ldots, 1+t_{m} \frac{x_{m}-1}{t_{m}}\right)=0 .
$$

This means that $f\left(x_{1}, x_{2}, \ldots, x_{m}\right)=0$, a contradiction. The proof is complete.

Recall that a generalized polynomial identity is a generalized Laurent polynomial identity in which all powers of indeterminates are non-negative.

Lemma 2.4. If $\mathrm{M}_{n}(D)$ satisfies a generalized polynomial identity, then $D$ is centrally finite, i.e. $D$ is a finite dimensional vector space over $F$.

Proof. This lemma is followed from [2, Theorem 6.1.9].

Now, we are ready to prove the main result of this work.

Theorem 2.5. Let $D$ be an algebraic division ring with uncountable center $F$ and $[D: F]=\infty$. If $N$ is an almost subnormal subgroup of $\mathrm{GL}_{n}(D)$ satisfying a GLPI $f\left(x_{1}, x_{2}, \ldots, x_{m}\right)=0$, then $N$ is central.

Proof. We first claim that if $\mathrm{GL}_{n}(D)$ satisfies a GLPI $g\left(x_{1}, x_{2}, \ldots, x_{m}\right)=0$ then $D$ is centrally finite. In fact, by Lemma 2.3 ,

$$
g\left(1+t_{1} y_{1}, 1+t_{2} y_{2}, \ldots, 1+t_{m} y_{m}\right) \neq 0 .
$$

Moreover,

$g\left(1+t_{1} y_{1}, 1+t_{2} y_{2}, \ldots, 1+t_{m} y_{m}\right)=\sum_{j_{1}, j_{2}, \ldots, j_{m} \geq 0} t_{1}^{j_{1}} t_{2}^{j_{2}} \cdots t_{m}^{j_{m}} p_{j_{1} j_{2} \ldots j_{m}}\left(y_{1}, y_{2}, \ldots, y_{m}\right)$ where $p_{j_{1} j_{2} \ldots j_{m}}$ are generalized polynomials over $\mathrm{M}_{n}(D)$ and

$$
p_{00 \ldots 0}=g(1,1, \ldots, 1)=0 .
$$

Thus, there exist $j_{1}^{*}, j_{2}^{*}, \ldots, j_{m}^{*}>0$ such that $p_{j_{1}^{*} j_{2}^{*} \ldots j_{m}^{*}}\left(y_{1}, y_{2}, \ldots, y_{m}\right) \neq 0$. Now, since $D$ is algebraic over its uncountable center $F$, by [1, Theorem 2.10], $\mathrm{M}_{n}(D)$ is algebraic over $F$. Let $c_{1}, c_{2}, \ldots c_{m}$ be arbitary elements in $\mathrm{M}_{n}(D)$, by Lemma 2.2, we have $1+\alpha_{1} c_{1}, 1+\alpha_{2} c_{2}, \ldots, 1+\alpha_{m} c_{m} \in \mathrm{GL}_{n}(D)$, for infinitely many elements $\alpha_{1}, \alpha_{2}, \ldots, \alpha_{m}$ in $F$. Hence

$$
g\left(1+\alpha_{1} c_{1}, 1+\alpha_{2} c_{2}, \ldots, 1+\alpha_{m} c_{m}\right)=0,
$$


for infinitely many elements $\alpha_{1}, \alpha_{2}, \ldots, \alpha_{m}$ in $F$. Due to Equation (1) in Lemma 2.2 , we can write $g\left(1+\alpha_{1} c_{1}, 1+\alpha_{2} c_{2}, \ldots, 1+\alpha_{m} c_{m}\right)=\frac{h\left(\alpha_{1}, \alpha_{2}, \ldots, \alpha_{m}\right)}{k\left(\alpha_{1}, \alpha_{2}, \ldots, \alpha_{m}\right)}$, where $h\left(t_{1}, t_{2}, \ldots, t_{m}\right) \in \mathrm{M}_{n}(D)\left[t_{1}, t_{2}, \ldots, t_{m}\right], k\left(t_{1}, t_{2}, \ldots, t_{m}\right) \in F\left[t_{1}, t_{2}, \ldots, t_{m}\right]$.

Since $g\left(1+\alpha_{1} c_{1}, 1+\alpha_{2} c_{2}, \ldots, 1+\alpha_{m} c_{m}\right)=0$, for infinitely many elements $\alpha_{1}, \alpha_{2}, \ldots, \alpha_{m}$ in $F$, it follows that $h\left(\alpha_{1}, \alpha_{2}, \ldots, \alpha_{m}\right)=0$ for infinitely many elements $\alpha_{1}, \alpha_{2}, \ldots, \alpha_{m}$ in $F$. By Lemma $2.1, h\left(t_{1}, t_{2}, \ldots, t_{m}\right)$ is identically zero, so is $g\left(1+t_{1} c_{1}, 1+t_{2} c_{2}, \ldots, 1+t_{m} c_{m}\right)$. Observe that

$g\left(1+t_{1} c_{1}, 1+t_{2} c_{2}, \ldots, 1+t_{m} c_{m}\right)=\sum_{j_{1}, j_{2}, \ldots, j_{m} \geq 0} t_{1}^{j_{1}} t_{2}^{j_{2}} \cdots t_{m}^{j_{m}} p_{j_{1} j_{2} \ldots j_{m}}\left(c_{1}, c_{2}, \ldots, c_{m}\right)$, so $p_{j_{1}^{*} j_{2}^{*} \ldots j_{m}^{*}}\left(c_{1}, c_{2}, \ldots, c_{m}\right)=0$. Thus, $p_{j_{1}^{*} j_{2}^{*} \ldots j_{m}^{*}}\left(y_{1}, y_{2}, \ldots, y_{m}\right)$ is a generalized polynomial identity of $\mathrm{M}_{n}(D)$. By Lemma $2.4, D$ is centrally finite. The claim is proved.

Now suppose that $N$ is non-central. We consider the following two cases.

Case 1. In the case when $n \geq 2$, by [12, Theorem 3.3], $N$ is a normal subgroup of $\mathrm{GL}_{n}(D)$. Fix an element $k \in N \backslash F$, and put

$$
g\left(y_{1}, \ldots, y_{m}\right)=f\left(y_{1} k y_{1}^{-1}, \ldots, y_{m} k y_{m}^{-1}\right) .
$$

Then, since $a k a^{-1} \in N$ for any $a \in \mathrm{GL}_{n}(D), g=0$ is a GLPI of $\mathrm{GL}_{n}(D)$. Hence, $D$ is centrally finite, a contradiction.

Case 2. In the case when $n=1$, suppose $N=N_{r} \leq N_{r-1} \leq \cdots \leq N_{1}=D^{*}$ is an almost normal series in $D^{*}$. We claim that if $N$ satisfies a GLPI, then so does $D^{*}$. It suffices to prove that $N_{r-1}$ satisfies a GLPI. In fact, if $N_{r}$ is normal in $N_{r-1}$, then by the same argument in Case 1 we get the claim. If $\left[N_{r-1}: N_{r}\right]=\ell<\infty$, then $a_{1}^{\ell !}, a_{2}^{\ell !}, \ldots, a_{m}^{\ell !} \in N_{r}$ for any $a_{1}, a_{2}, \ldots, a_{m} \in N_{r-1}$. Hence, $f\left(a_{1}^{\ell !}, a_{2}^{\ell !}, \ldots, a_{m}^{\ell !}\right)=0$. Thus $g\left(x_{1}, x_{2}, \ldots, x_{m}\right)=f\left(x_{1}^{\ell !}, x_{2}^{\ell !}, \ldots, x_{m}^{\ell !}\right)$ is a GLPI of $N_{r-1}$. The claim is proved. Therefore, $D$ is centrally finite, a contradiction.

Thus, the proof is now complete.

Next, we prove some results for maximal subgroups in $\mathrm{GL}_{n}(D)$.

Theorem 2.6. Let $D$ be a division ring with infinite center $F$ and $[D: F]=\infty$. Assume that $M$ is a maximal subgroup of $\mathrm{GL}_{n}(D)$ such that $F[M]$ is algebraic over $F$, where $F[M]$ is the $F$-subalgebra of $\mathrm{M}_{n}(D)$ generated by $M$ over $F$. If $M$ satisfies an LPI, then $M$ is absolutely irreducible.

Proof. By the maximality of $M$, either $F[M]^{*}=M$ or $F[M]^{*}=\mathrm{GL}_{n}(D)$. We show that the first case can not occur. Indeed, by hypothesis $M$ satisfies an LPI, so $F[M]^{*}$ satisfies an LPI. Using the technique in the first part of the proof of 
Theorem 2.5, we can prove that $F[M]$ satisfies a polynomial identity. Therefore, by [10, Theorem 3.5], $D$ is centrally finite, a contradiction.

Thus, we conclude $F[M]^{*}=\mathrm{GL}_{n}(D)$ and $F[M]=\mathrm{M}_{n}(D)$. Hence $M$ is absolutely irreducible.

Corollary 2.7. Let $D$ be a division ring with infinite center $F$. Assume that $M$ is a maximal subgroup of $\mathrm{GL}_{n}(D)$ such that $F[M]$ is algebraic over $F$. If $M$ satisfies a group identity, then $D$ is centrally finite or $M$ is absolutely irreducible.

Lemma 2.8. Let $G$ be a group and $N$ be an almost subnormal subgroup of $G$. For any subgroup $H$ of $G$, the subgroup $H \cap N$ is an almost subnormal subgroup of $H$.

Proof. The proof is elementary.

Lemma 2.9. Let $D$ be a division ring with infinite center $F$. If $D^{*}$ contains a non-central almost subnormal subgroup which satisfies an LPI $f=0$, then $D$ is centrally finite.

Proof. This lemma is from [7, Theorem 1.1].

Theorem 2.10. Let $D$ be a division ring with infinite center $F$ and $[D: F]=\infty$. Let $N$ be a non-central almost subnormal subgroup of $D^{*}$. Suppose that $M$ is a maximal subgroup of $N$ such that $\mathrm{C}_{D}(M) \backslash F$ contains an algebraic element over $F$. If $M$ satisfies an LPI, then $M$ is abelian.

Proof. This proof is a slight modification of the one of [9, Theorem 2]. Suppose that $\alpha \in \mathrm{C}_{D}(M) \backslash F$ is algebraic over $F$. Put $L:=F(\alpha)$ and $B:=\mathrm{C}_{D}(L)$. Then, $[L: F]<\infty$. By the Double Centralizer Theorem, $B$ is a division ring with center $L$. Since $\alpha \in \mathrm{C}_{D}(M)$, we have $M \leq B^{*}$. Therefore, $M \leq N \cap B^{*} \leq N$. By the maximality of $M$ in $N$, we have $N \cap B^{*}=N$ or $N \cap B^{*}=M$. The first case can not occur. To prove this, we claim that $N \nsubseteq B^{*}$. Suppose that $N \subseteq B^{*}$. Then, $F(N) \subseteq B$. Since $N$ normalizes $F(N)$, by [3, Theorem 1], we have $F(N)=D$ and consequently $B=D$. This contradicts the fact that $\alpha$ is not in $F$. Hence $N \nsubseteq B^{*}$ and $N \cap B^{*}=M$. By Lemma 2.8, we have $B^{*} \cap N$ is an almost subnormal subgroup of $B^{*}$. Thus, $M$ is an almost subnormal subgroup of $B^{*}=\mathrm{C}_{D}(F(\alpha))^{*}$.

Now, suppose that $M$ is non-abelian. By [12, Corollary 2.3], $M$ is a non-central almost subnormal subgroup of $B^{*}$. Since $M$ satisfies an LPI, by Lemma 2.9, we have $[B: L]<\infty$. Recall that $[L: F]=r<\infty$, so $[B: F]<\infty$. By part (ii) of $[6$, Centralizer Theorem, p. 42], we have

$$
D \otimes_{F} L \cong \mathrm{M}_{r}\left(B \otimes_{L} \mathrm{C}_{D}(B)\right) \cong \mathrm{M}_{r}\left(B \otimes_{L} \mathrm{C}_{D}\left(\mathrm{C}_{D}(L)\right)\right) \cong \mathrm{M}_{r}\left(B \otimes_{L} L\right) \cong \mathrm{M}_{r}(B) .
$$


Since $\mathrm{M}_{r}(B)$ is a finite dimensional vector space over $F$, we conclude that $[D: F]<$ $\infty$, a contradiction. The proof is complete.

Acknowledgement. The author would like to thank the referees for the useful suggestions and comments. This research is funded by Vietnam National University Ho Chi Minh City (VNU-HCM) under grant number B2016-18-01.

\section{References}

[1] S. Akbari and M. Arian-Nejad, Left Artinian algebraic algebras, Algebra Colloq., 8(4) (2001), 463-470.

[2] K. I. Beidar, W. S. Martindale 3rd and A. V. Mikhalev, Rings with Generalized Identities, Monographs and Textbooks in Pure and Applied Mathematics, 196, Marcel Dekker, Inc., New York, 1996.

[3] M. H. Bien, T. T. Deo and B. X. Hai, On a division subring normalized by an almost subnormal subgroup in division rings, arXiv:1801.01271.

[4] M. H. Bien, D. Kiani and M. Ramezan-Nassab, Some skew linear groups satisfying generalized group identities, Comm. Algebra, 44(6) (2016), 2362-2367.

[5] M. A. Dokuchaev and J. Z. Gonçalves, Identities on units of algebraic algebras, J. Algebra, 250(2) (2002), 638-646.

[6] P. K. Draxl, Skew Fields, London Mathematical Society Lecture Note Series, 81, Cambridge University Press, Cambridge, 1983.

[7] B. X. Hai, T. H. Dung and M. H. Bien, Almost subnormal subgroups in division rings with generalized algebraic rational identities, arXiv:1709.04774.

[8] B. Hartley, Free groups in normal subgroups of unit groups and arithmetic groups, Representation theory, group rings, and coding theory, Contemp. Math., 93, Amer. Math. Soc., Providence, RI, (1989), 173-177.

[9] D. Kiani, Polynomial identities and maximal subgroups of skew linear groups, Manuscripta Math., 124(2) (2007), 269-274.

[10] D. Kiani and M. Mahdavi-Hezavehi, Identities on maximal subgroups of $\mathrm{GL}_{n}(D)$, Algebra Colloq., 12(3) (2005), 461-470.

[11] L. Makar-Limanov, On subnormal subgroups of skew fields, J. Algebra, 114(2) (1988), 261-267.

[12] N. K. Ngoc, M. H. Bien and B. X. Hai, Free subgroups in almost subnormal subgroups of general skew linear groups, St. Petersburg Math. J., 28(5) (2017), 707-717.

[13] M. Ramezan-Nassab and D. Kiani, Nilpotent and polycyclic-by-finite maximal subgroups of skew linear groups, J. Algebra, 399 (2014), 269-276. 
[14] L. H. Rowen, Polynomial Identities in Ring Theory, Pure and Applied Mathematics, 84, Academic Press, Inc. [Harcourt Brace Jovanovich, Publishers], New York-London, 1980.

\section{Truong Huu Dung}

VNUHCM - University of Science

Faculty of Mathematics and Computer Science

227 Nguyen Van Cu Str., Dist. 5, HCM-City, Vietnam

and

Dong Nai University

4 Le Quy Don Str., Tan Hiep Ward, Bien Hoa City

Dong Nai Province, Vietnam

e-mail: thdung@dnpu.edu.vn or dungth0406@gmail.com 